\title{
SISTEMA DE PREPARO DO SOLO E SUA INFLUÊNCIA NA ATIVIDADE
} MICROBIANA

Doi:http://dx.doi.org/10.1590/1809-4430-Eng.Agric.v35n3p 506-513/2015

\section{JULIANA P. DADALTO ${ }^{1}$, HAROLDO C. FERNANDES ${ }^{2}$, MAURI M. TEIXEIRA ${ }^{3}$, PAULO R. CECON ${ }^{4}$, ANTONIO T. DE MATOS ${ }^{5}$.}

\begin{abstract}
RESUMO: O sistema de preparo mecanizado do solo influencia diretamente sobre as propriedades físicas e biológicas do solo, além de propiciar ambiente adequado para o estabelecimento das culturas. O preparo do solo pode interferir na atividade microbiológica. Dessa forma, objetivou-se com o presente trabalho avaliar como os sistemas de preparo mecanizado do solo podem afetar sua atividade microbiológica. Os tratamentos foram os seguintes tipos de preparo do solo: plantio direto (PD), plantio convencional (PC) e cultivo mínimo (CM). Todas as análises foram feitas em três instantes: antes do preparo do solo, depois do preparo e 14 dias após o preparo do solo. Os tratamentos foram dispostos no delineamento em blocos casualizados, com quatro blocos. As coletas foram realizadas em triplicata, em duas faixas: 0,0 a $0,10 \mathrm{~m}$ e de 0,10 a $0,20 \mathrm{~m}$ de profundidade. Foram determinados o carbono orgânico total (COT), o carbono da biomassa microbiana (CBM), a respiração basal do solo (RBS), o quociente metabólico $\left(\mathrm{qCO}_{2}\right)$ e o quociente microbiano (qMic). O plantio direto apresentou maiores índices de carbono da biomassa microbiana e da respiração basal do solo, na faixa de $0,0-0,10 \mathrm{~m}$ de profundidade. $O$ plantio direto foi o sistema que apresentou menor interferência da atividade microbiológica do solo.
\end{abstract}

PALAVRAS-CHAVE: plantio direto, microrganismos, carbono orgânico.

\section{TILAGE INFLUENCE ON SOIL MICROBIAL ACTIVITY}

\begin{abstract}
In addition to provide appropriate environment for crops, mechanized tillage directly affects physical and biological soil properties; besides influencing microbial activity. Therefore, we aimed at assessing how different mechanized tillage systems may affect soil microbiological activities. Treatments consisted of the following soil tillage types: No-tillage (PD), conventional tillage (PC) and minimum tillage $(\mathrm{CM})$. Treatments were arranged in a randomized block design. All analyses were performed at three moments: before and after soil preparation and 14 days after tillage. Collections were made in triplicate at two soil depths $(0-0.10 \mathrm{~m}$ and $0.10-$ $0.20 \mathrm{~m}$ ). It was determined values of total organic carbon (COT), microbial biomass carbon (CBM), basal soil respiration (RBS), metabolic quotient $\left(\mathrm{qCO}_{2}\right)$ and microbial quotient (qMic). No-tillage system showed higher ratios of microbial biomass carbon and soil basal respiration at $0.0-0.10 \mathrm{~m}$ depth. Therefore, no till was the system that had greater microbial activity in the soil.
\end{abstract}

KEYWORDS: tillage, microorganisms, organic carbon

\section{INTRODUÇÃO}

A evolução da agricultura está intimamente ligada à mecanização das operações do campo. A mecanização torna o trabalho agrícola menos árduo, a lavoura mais homogênea e aumenta a produtividade das culturas. Um dos obstáculos da mecanização agrícola é o aumento da compactação do solo devido ao tráfego de máquinas (ROBOREDO et al, 2010). O sistema de

\footnotetext{
${ }^{1}$ Eng $^{\mathrm{a}}$ Agrícola e Ambiental, Doutoranda, Departamento de Engenharia A grícola, UFV/Viçosa - MG,(31) 3899-1860, juliana.dadalto@ufv.br

${ }^{2}$ Eng $^{\circ}$ A grícola, Prof. Doutor, Departamento de Engenharia Agrícola, UFV/Viçosa-MG, (31) 3899-1860, haroldo@ufv.br

${ }^{3}$ Eng $^{\circ}$ A grônomo, Prof. Doutor, Departamento de Engenharia Agrícola, UFV/Viçosa-MG, (31) 3899-1878, mauri@ufv.br

${ }^{4}$ Eng $^{\circ}$ A grônomo, Prof. Doutor, Departamento de Estatística, UFV/Viçosa-MG, (31) 3899-1781, cecon@ufv.br

${ }^{5}$ Eng $^{\circ}$ A grícola, Prof. Doutor, Departamento de Engenharia Agrícola, UFV/Viçosa -MG, (31) 3899-1886, atmatos@ufv.br Recebido pelo Conselho Editorial em: 29-8-2014
} 
preparo mecanizado do solo influencia diretamente sobre as propriedades físicas e biológicas do solo, além de propiciar ambiente para o estabelecimento das culturas. O tipo de preparo do solo, assim como o estabelecimento de nova cultura, impõe ao sistema solo/microrganismos nova condição que influencia diretamente sobre a disponibilidade, a qualidade e a quantidade dos nutrientes no solo (LISBOA et al., 2012). Segundo ISLAM e WEIL (2000), a atividade microbiana está relacionada à degradação ou à qualidade do solo, sendo que as análises de carbono da biomassa microbiana e a relação da respiração basal por unidade de biomassa microbiana são indicadores para esta determinação; além disso, a atividade microbiana do solo pode ser mensurada por meio do quociente microbiano.

No plantio convencional, o revolvimento do solo permite o rompimento dos agregados, além de promover maior contato entre os resíduos vegetais e o solo. Esses fatores estimulam, temporariamente, a microbiota a degradar a matéria orgânica do solo (LISBOA et al., 2012). A respiração basal do solo mede a atividade microbiológica do solo onde os microrganismos degradam os compostos orgânicos a $\mathrm{CO}_{2}$ (SILVA et al., 2013). Assim como a matéria orgânica, a umidade diminui à medida que se aumenta a profundidade. Em solos com presença de palhada, as perdas por evaporação são menores em comparação com solos sem cobertura ve getal, promovendo um ambiente mais adequado ao estabelecimento da cultura (PERES et al., 2010).

Os estados fisiológicos das células microbianas influenciam na respiração basal do solo, assim com a umidade, a temperatura, a estrutura do solo, a textura, a quantidade de matéria orgânica, dentre outros (SILVA et al., 2010). A análise isolada do carbono da biomassa microbiana $(\mathrm{CBM})$ e da respiração basal do solo (RBS) pode limitar a análise do solo quanto à atividade microbiana; assim, o quociente metabólico, junto com essas variáveis fornecem informações mais adequadas para o entendimento da atividade microbiológica do solo (ALVES et al., 2011).

$\mathrm{O}$ quociente metabólico $\left(\mathrm{qCO}_{2}\right)$ é a razão entre a respiração basal e a biomassa microbiana do solo, por unidade de tempo (ANDERSON; DOMSCH, 1993). Ele expressa quanto de $\mathrm{CO}_{2}$ é liberado pela biomassa microbiana em função do tempo, representando a taxa de respiração específica da biomassa microbiana (ALVES et al., 2011). O quociente microbiano (qMic) representa a relação entre o carbono da biomassa microbiana e o carbono orgânico total. Essa relação tem sido utilizada como indicador da qualidade da matéria orgânica do solo, indicando a quantidade de carbono orgânico que está imobilizado na biomassa e demonstrando, ainda, a eficiência dos microrganismos na utilização dos compostos orgânicos (SILVA et al., 2010).

O qMic reflete o percentual de reserva do carbono orgânico total no solo, e pode-se perceber que áreas com baixa atividade microbiana apresentam baixos valores de quociente microbiano, indicando menor reserva de compostos orgânicos nessas áreas (C ARNEIRO et al., 2009). Altos índices de qMic indicam que a matéria orgânica do solo é ativa e es tá sujeita a ser decomposta pela microbiota. Dessa forma, objetivou-se com o presente trabalho avaliar como os diferentes sistemas de preparo mecanizado do solo podem afetar sua atividade microbioló gica.

\section{MATERIAL E MÉTODOS}

$\mathrm{O}$ experimento foi realizado numa área experimental pertencente à Universidade Federal de Viçosa - Minas Gerais, coordenadas $20^{\circ} 45^{\prime}$ '16,9" latitude sul e $42^{\circ} 50$ ' 22,6" longitude oeste, com altitude de $648 \mathrm{~m}$. A região apresenta clima montanhoso, e seu clima é classificado, segundo Köppen (1948), como mesotérmico úmido.

Para a realização do experimento, utilizou-se dos seguintes tipos de preparo do solo como tratamentos: plantio direto (PD), preparo convencional (PC) e cultivo mínimo (CM). O preparo convencional foi estabelecido por uma aração e duas gradagens; o cultivo mínimo, por uma passada com escarificador; e o plantio direto, por uma passada com a semeadora adubadora de plantio direto. Todas as análises foram feitas em três épocas de amostragem, antes do preparo do solo, depois do preparo e 14 dias após o preparo do solo, em duas profundidades: 0,0 a $0,10 \mathrm{~m}$ e de 0,10 a $0,20 \mathrm{~m}$. As amostras realizadas depois do preparo do solo foram coletadas, aproximadamente, 3 horas após o preparo. $\mathrm{O}$ experimento foi disposto em esquema de parcelas subsubdivididas, tendo 
nas parcelas os tipos de preparo do solo; nas subparcelas, as profundidades; e nas subsubparcelas, as épocas de amostragem, no delineamento de blocos casualizados, com quatro repetições.

As análises microbiológicas foram realizadas no Laboratório de Matéria Orgânica, do departamento de solos da Universidade Federal de Viçosa. As coletas foram realizadas, utilizando o trado holandês, sendo coletadas três amostras simples, que formaram uma amostra composta, para cada profundidade, em cada parcela. As amostras compostas foram peneiradas em peneira com 2 mm de malha, acondicionadas em sacos plásticos, identificadas e guardadas em caixas térmicas até à chegada ao laboratório, onde foram transferidas para geladeira, permanecendo em média a $4{ }^{\circ} \mathrm{C}$ até ao momento das análises.

\section{Carbono Orgânico Total (COT)}

O carbono orgânico total (COT) foi determinado por meio da oxidação via úmida segundo metodologia de MENDONÇA \& MATOS (2005). Pesou-se 0,2 g de terra fina seca ao ar (TFSA), que foram transferidos para tubos de digestão. Adicionaram-se $5 \mathrm{~mL}$ de solução de dicromato de potássio e 7,5 mL de ácido sulfúrico concentrado aos tubos de digestão. Essa solução foi levada ao bloco digestor a $170{ }^{\circ} \mathrm{C}$ por 30 minutos. Após a digestão, as soluções foram transferidas para o erlenmeyer, onde foram adicionadas água destilada e solução indicadora. A solução final foi titulada com solução de sulfato ferroso amoniacal a $0,2 \mathrm{~mol} \mathrm{L^{-1 }}$. Para as mesmas condições, foram realizados seis brancos, sendo que três foram para digestão e os demais não foram aquecidos. $\mathrm{O}$ valor do COT foi calculado com base no volume de sulfato ferroso amoniacal gasto na titulação dos brancos e da amostra. A partir da [eq. (1)], pode-se determinar a quantidade de carbono orgânico total da amostra em dag kg-1.

$$
\mathrm{COT}=\frac{A \times \mathrm{MSF} \times 3 \times 100}{\mathrm{~Pa}}
$$

em que,

A - Volume calculado $(\mathrm{mL})$;

MSF - Molaridade do Sulfato Ferroso $\left(\mathrm{mol} \mathrm{L}^{-1}\right)$, e

$\mathrm{Pa}$ - Peso da amostra (mg).

\section{Carbono da Biomassa Microbiana}

A determinação do carbono da biomassa microbiana (CBM) foi feita segundo metodologia de MENDONÇA \& MATOS (2005). Foram pesados $20 \mathrm{~g}$ de solo em placas de Petri e outros $20 \mathrm{~g}$ de solo em erlenmeyer. O solo das placas de Petri foi irradiado no micro-ondas e, posteriormente, transferido para erlenmeyer. Ao solo foi adicionada solução extratora (sulfato de potássio) e agitada por 30 minutos em agitador horizontal, e filtrou-se o sobrenadante com auxílio de filtro quantitativo lento. Após a extração, foram pipetados $10 \mathrm{~mL}$ do extrato filtrado para um erlenmeyer, e foram adicionados $2 \mathrm{~mL}$ de dicromato de potássio a $0,066 \mathrm{~mol} \mathrm{~L}^{-1}, 10 \mathrm{~mL}$ de ácido sulfúrico e $50 \mathrm{~mL}$ de água destilada. Após a solução esfriar, foram adicionadas três gotas de solução indicadora e titulouse o excesso do dicromato com sulfato ferroso amoniacal a $0,03 \mathrm{~mol} \mathrm{~L}^{-1}$. Foram feitos seis brancos, três com solução extratora e os demais reagentes, e três sem a solução extratora. Calculando-se a diferença do carbono nas amostras irradiadas e não irradiadas, determinou-se o carbono da biomassa microbiana em $\mu \mathrm{g} \mathrm{g}^{-1}$ de $\mathrm{C}$ no solo.

\section{Respiração Basal do Solo}

Foi determinada pelo método estático sugerido por MENDONÇA \& MATOS (2005). As amostras do solo foram incubadas junto com solução de $\mathrm{NaOH}$ a $0,5 \mathrm{~mol} \mathrm{~L}^{-1}$, em recipiente fechado hermeticamente a $25{ }^{\circ} \mathrm{C}$. Após sete dias, o recipiente foi aberto por 15 minutos, para troca de ar com o ambiente. Foram pipetados $10 \mathrm{~mL}$ da solução de $\mathrm{NaOH}$ incubada em erlenmeyer, e adicionaram-se $10 \mathrm{~mL}$ de cloreto de bário a $0,05 \mathrm{~mol} \mathrm{~L}^{-1}$. A solução do erlenmeyer foi titulada com $\mathrm{HCl}$ a $0,25 \mathrm{~mol} \mathrm{~L}^{-1}$ após a adição do indicador (fenolftaleína). A respiração do solo foi determinada em mg de carbono respirado em $100 \mathrm{~cm}^{3}$ de solo. 


\section{Quociente Metabólico (qCO 2$)$}

Foi determinado a partir da respiração basal do solo e pelo carbono da biomassa microbiana, conforme a [eq. (2)]. Sua unidade é expressa em mg C-CO $\mathrm{Cg}_{2}^{-1} \mathrm{CO}_{2} \mathrm{dia}^{-1}$ (ANDERSON; DOMSCH, 1993).

$$
\mathrm{qCO}_{2}=\frac{\mathrm{RBS}}{\mathrm{CBM}}
$$

em que,

RBS - Taxa de respiração basal do solo (mg de C-CO $\left.\mathrm{Cg}^{-1} \mathrm{dia}^{-1}\right)$, e

$\mathrm{CBM}$ - Carbono da biomassa microbiana ( $\mathrm{mg} \mathrm{de} \mathrm{CO}_{2} \mathrm{~kg}^{-1}$ ).

\section{Quociente Microbiano (qMic)}

Foi determinado pela relação entre o carbono da biomassa microbiana $(\mathrm{CBM})$ e o carbono orgânico total (COT), conforme a [eq. (3)] (ANDERSON; DOMSCH, 1993), sendo expresso em porcentagem.

$$
\mathrm{qMi}=\frac{\mathrm{CBM}}{\mathrm{COT}}
$$

em que,

CBM - Carbono da biomassa microbiana $\left(\mathrm{mg} \mathrm{kg}^{-1}\right), \mathrm{e}$

COT - Carbono orgânico total $\left(\mathrm{mg} \mathrm{kg}^{-1}\right)$.

\section{Análises estatísticas}

Os dados foram analisados por meio da análise de variância, e as médias comparadas utilizando o teste de Tukey, adotando o nível de 5\% de probabilidade. Todas as análises foram realizadas com o auxílio do software estatístico SAEG (UFV, 2007).

\section{RESULTADOS E DISCUSSÃO}

\section{Carbono Orgânico Total}

Houve diferença estatística nas médias do carbono da biomassa microbiana em relação à profundidade e ao tipo de sistema de preparo. Com o aumento da profundidade, observou-se decréscimo no valor do COT, passando de 21,95 $\mathrm{g} \mathrm{kg}^{-1}$ na faixa de $0,0-0,10 \mathrm{~m}$ de profundidade, para $18,58 \mathrm{~g} \mathrm{~kg}^{-1}$ na faixa de $0,10-0,20 \mathrm{~m}$ de profundidade. Avaliando a quantidade de carbono em diferentes sistemas de preparo do solo e épocas de amostragem, LOSS et al. (2010) encontraram valores de carbono orgânico total de 13,7 e $11,5 \mathrm{~g} \mathrm{~kg}^{-1}$, nas profundidades de 0,0-0,05 e 0,05 $0,10 \mathrm{~m}$, respectivamente. Em relação aos tipos de sistemas de preparo do solo, não houve diferença estatística entre o plantio direto e o sistema de plantio convencional. Já o cultivo mínimo apresentou diferença estatística dos demais tratamentos a $1 \%$ de probabilidade. As maiores médias de COT foram encontradas no sistema de plantio direto seguido do preparo convencional e, finalmente, o cultivo mínimo, sendo de $23,81 \mathrm{~g} \mathrm{~kg}^{-1}, 22,24 \mathrm{~g} \mathrm{~kg}^{-1}$ e $14,74 \mathrm{~g} \mathrm{~kg}^{-1}$, respectivamente.

FONTANA et al. (2011), ao estudarem os compartimentos da matéria orgânica em solo com diferentes coberturas, encontraram menores valores de COT em maiores profundidades, além de maiores teores de COT em mata nativa, em comparação a solos cultivados. Esses valores podem estar associados à maior reserva e ao aporte de matéria orgânica nos solos da mata, além da menor ação do homem.

FERNANDES et al. (2013) avaliaram o carbono orgânico total em áreas revegetadas e áreas desertificadas e encontraram diferenças estatísticas entre as áreas, sendo que a área revegetada apresentou maiores índices de COT, após um ano do plantio. 


\section{Carbono da B iomassa Microbiana}

Para o carbono da biomassa microbiana, houve interação significativa entre o sistema de preparo em função da profundidade. Na faixa de 0,0-0,10 $\mathrm{m}$ de profundidade, o sistema de plantio direto não diferiu estatisticamente do cultivo mínimo, e o preparo convencional foi igual, estatisticamente, ao cultivo mínimo. Para essa profundidade, as maiores médias foram encontradas no plantio direto, seguidas pelo cultivo mínimo e, finalmente, no plantio convencional, sendo de $667,98 \mathrm{mg} \mathrm{kg}^{-1}, 533,65 \mathrm{mg} \mathrm{kg}^{-1}$ e 443,39 $\mathrm{mg} \mathrm{kg}^{-1}$, respectivamente. Já na faixa de 0,10 a 0,20 m de profundidade, não houve diferença estatística a $5 \%$ de probabilidade, pelo teste de Tukey.

Ao estudarem o efeito do tipo de manejo nos atributos do solo, LOURENTE et al. (2011) não encontraram diferença estatística nos valores de carbono da biomassa microbiana entre o sistema de preparo convencional e o plantio direto. Fato esse associado à coleta de amostras ser realizada no primeiro ano do preparo convencional. Ao avaliar os atributos biológicos como indicadores das alterações sofridas no solo, NEVES et al. (2009) encontraram valores de CBM para o cerrado nativo da ordem de $1.498 \mu \mathrm{m} \mathrm{g}^{-1}$, nas camadas superficiais. Embora o efeito da profundidade não tenha significância estatisticamente, há uma tendência de o valor do carbono da biomassa microbiana diminuir com o aumento da profundidade.

\section{Respiração Basal do Solo}

Para a respiração basal do solo, não houve diferenças estatísticas a 5\% de probabilidade, em relação ao tipo de preparo, profundidade e tempo de amostragem. As médias dos valores da respiração basal do solo (RBS) encontram-se na Tabela 1.

TABELA 1. Valores médios de respiração basal do solo $\left(\mathrm{mg} \mathrm{kg}^{-1}\right)$ em função do sistema de preparo e da profundidade do solo $(\mathrm{m})$ e tempo de amostragem. Basal soil respiration means $\left(\mathrm{mg} \mathrm{kg}^{-1}\right)$ as a function of soil tillage system, depth (m) and sampling time.

\begin{tabular}{ccccccc}
\hline Sistema de & \multicolumn{2}{c}{ Antes } & \multicolumn{2}{c}{ Depois } & \multicolumn{2}{c}{14 dias depois } \\
\cline { 2 - 6 } Preparo & $0,0-0,10$ & $0,10-0,20$ & $0,0-0,10$ & $0,10-0,20$ & $0,0-0,10$ & $0,10-0,20$ \\
\hline PD & 1011,89 & 1689,77 & 2739,17 & 1343,93 & 1517,56 & 1129,92 \\
PC & 1172,18 & 1088,76 & 2317,20 & 1043,63 & 1311,16 & 1347,02 \\
CM & 1048,07 & 887,26 & 795,96 & 874,42 & 2030,38 & 1101,14 \\
\hline CV $(\%)$ & 5,86 & 25,52 & 39,46 & 15,73 & 16.9 & 8,62 \\
\hline
\end{tabular}

Legenda: PD - Plantio direto; PC - Preparo convencional; CM - cultivo mínimo.

ALVES et al. (2011) estudaram a influência dos diversos sistemas na atividade microbiana e não observaram diferenças estatísticas em relação à respiração basal do solo nos tipos de manejo do solo, integração lavoura-agropecuária, vegetação nativa e vegetação nativa em recuperação.

$\mathrm{O}$ aumento dos valores de respiração basal após o preparo do solo está relacionado às perturbações que o solo e as populações microbianas sofrem (NASCIMENTO et al., 2009). Porém, segundo ISLAM \& WEIL (2000), altas taxas de respiração podem indicar ou não algum tipo de distúrbio no solo; por esse motivo, a análise da respiração basal não deve ser feita isoladamente e, sim, em conjunto, por meio do quociente metabólico.

\section{Quociente Metabólico}

Para o quociente metabólico $\left(\mathrm{qCO}_{2}\right)$, observa-se que houve diferença estatística somente antes do preparo do solo, o plantio direto não apresentou diferença estatística do cultivo mínimo, mas apresentou diferença estatística do preparo convencional (Tabela 2). Após o preparo do solo, os valores encontrados, para todos os tipos de preparo, não apresentaram diferença estatística, provavelmente, isso se deve ao fato de que as coletas das amostras foram realizadas logo após a passada do implemento no solo. O plantio convencional apresentou um valor alto após 14 dias do preparo do solo $\left(7,37 \mathrm{mg} \mathrm{kg}^{-1} \mathrm{dia}^{-1}\right)$, indicando que, nesse sistema de preparo, encontra-se em estresse. 
TABELA 2. Médias do $\mathrm{qCO}_{2}\left(\mathrm{mg} \mathrm{kg}^{-1} \mathrm{dia}^{-1}\right)$ em função do tipo de preparo e da época de amostragem. Means of $\mathrm{qCO}_{2}\left(\mathrm{mg} \mathrm{kg}^{-1} \mathrm{day}^{-1}\right)$ as function of soil tillage system and sampling time.

\begin{tabular}{cccc}
\hline Sistemas de & \multicolumn{3}{c}{ Tempo } \\
\cline { 2 - 4 } Preparo & Antes & Depois & 14 Dias \\
\hline PD & $7,41 \mathrm{a} \mathrm{A}$ & 4,24 a A & 3,81 a A \\
PC & $2,55 \mathrm{a} \mathrm{B}$ & $4,02 \mathrm{ab} \mathrm{A}$ & $7,37 \mathrm{~b} \mathrm{~A}$ \\
CM & $2,99 \mathrm{a} \mathrm{AB}$ & $1,95 \mathrm{a} \mathrm{A}$ & $3,82 \mathrm{a} \mathrm{A}$ \\
\hline CV $(\%)$ & 47,77 & 28,46 & 31,60 \\
\hline
\end{tabular}

Médias seguidas de mesma letra, maiúscula na coluna e minúscula na linha, não diferem entre si, pelo teste de Tukey, a 5\% de probabilidade. Legenda: PD - Plantio direto; PC - Preparo convencional; CM - cultivo mínimo.

Quando a biomassa microbiana se torna mais eficiente, menos $\mathrm{CO}_{2}$ é perdido para a atmosfera e maior taxa de carbono é incorporada à biomassa microbiana, resultando em menores valores de $\mathrm{qCO}_{2}$ (CUNHA et al., 2011). A substituição da vegetação acelera a decomposição dos resíduos vegetais e, assim, o valor do quociente metabólico aumenta (SILVA et al., 2007 ), explicando, assim, o aumento do quociente metabólico após o preparo do solo no sistema de plantio convencional.

\section{Quociente Microbiano}

O quociente microbiano foi significativo a $5 \%$ de probabilidade em relação ao tempo de preparo do solo (Tabela 3). Antes do preparo do solo, as médias foram estatisticamente iguais a depois do preparo do solo. Possivelmente, o valor do quociente microbiano, depois do preparo do solo, não sofreu grandes alterações devido às coletas do solo que foram realizadas imediatamente após o preparo do solo. Valores de quociente microbiano inferiores a $1 \%$ indicam que existe algum fator limitante à atividade microbiológica no solo (JAKELAITIS et al., 2008).

TABELA 3. Médias do qMic (\%) em função do tempo de preparo do solo. Means of qMic (\%) with regards of tillage timing.

\begin{tabular}{cc}
\hline Tempo & qMic \\
\hline Antes & $2,12 \mathrm{a}$ \\
Depois & $2,57 \mathrm{ab}$ \\
14 dias depois & $5,48 \mathrm{~b}$ \\
\hline CV $(\%)$ & 41,10
\end{tabular}

Médias seguidas de mesma letra não diferem entre si, pelo teste de Tukey, a 5\% de probabilidade.

Após 14 dias do preparo, a média do quociente microbiano foi diferente estatisticamente de antes do preparo do solo. Depois de 14 dias do preparo do solo, ocorreu o maior índice de qMic $(5,48 \%)$ em relação aos outros tempos de preparo. Esse valor indica que ocorreu maior incorporação do carbono orgânico do solo na biomassa microbiana. Ou seja, os microrganismos imobilizaram o carbono que estava disponível no solo em suas células. Segundo SILVA et al. (2010), o quociente microbiano indica a quantidade de carbono do solo que está imobilizado na biomassa microbiana. Também encontraram maiores valores de qMic para o cerrado nativo e menores valores para o preparo convencional.

\section{CONCLUSÕES}

O plantio direto apresentou maiores índices de carbono da biomassa microbiana na faixa de 0,0 - 0,10 m de profundidade, com $667,98 \mathrm{mg} \mathrm{kg}^{-1}$. O plantio convencional apresentou maiores valores de quociente metabólico após 14 dias do preparo do solo, com 7,37 mg kg $\mathrm{dia}^{-1}$. O plantio direto foi o sistema que apresentou menor interferência da atividade microbiológica do solo. 


\section{REFERÊNCIAS}

ALVES, T. D. S.; CAMPOS, L. L.; ElIAS NETO, N.; MATSUOKA, M.; LOUREIRO, M. F. Biomassa e atividade microbiana de solo sob vegetação nativa e diferentes sistemas de manejos. Acta Scientiarum. Agronomy, Maringá, v. 33, n. 2, 2011.

ANDERSON, T. H.; DOMSCH, K. H.The metabolic quotient for $\mathrm{CO}_{2}\left(\mathrm{qCO}_{2}\right)$ as a specific activiy parameter to assess the efect of environmental condition, such as $\mathrm{pH}$ on the microbial biomass of forest soils. Soil Biology and Bioche mistry, Amsterdam, v. 23, n. 3, p. 393-395, 1993.

CARNEIRO, M. A. C.; SOUZA, E. D. D.; REIS, E. F. D.; PEREIRA, H. S.; AZEVEDO, W. R. D. Atributos físicos, químicos e biológicos de solo de cerrado sob diferentes sistemas de uso e manejo. Revista Brasileira de Ciência do Solo, Viçosa, MG, v. 33, p. 147-157, 2009.

CUNHA, E. D. Q.; STONE, L. F.; FERREIRA, E. P. D. B.; DIDONET, A. D.; MOREIRA, J. A. A.; LEANDRO, W. M. Sistemas de preparo do solo e culturas de cobertura na produção orgânica de feijão e milho: II - atributos biológicos do solo. Revista Brasileira de Ciência do Solo, Viçosa, MG, v. 35, p. 603-611, 2011.

FERNANDES, M. M.; SILVE, M. D.; VELOSO, M. E. D. C.; OLIVEIRA, T. M.; FERNANDES, M. R. D. M.; SAMPAIO, F. M. T. Biomassa microbiana e matéria orgânica em áreas desertificadas revegetadas com pinhão-manso solteiro e consorciado com gramínea no sul do Piauí. Revista Brasileira de Ciências Agrárias, Recife, v. 8, n. 3, p. 464-469,2013.

FONTANA, A.; SILVA, C. F. D.; PEREIRA, M. G.; BRITO, R. J. D.; BENITES, V. D. M. Avaliação dos compartimentos da matéria orgânica em área de Mata Atlântica. Acta Scientiarum. Agronomy, Maringá, v. 33, n. 3, p. 545-550, 2011.

ISLAM, K. R.; WEIL, R. R. Land use effects on soil quality in a tropical forest ecosystem of Bangladesh. Agriculture Ecosystems and Environment, Amsterdam, v. 79, n. 1, p. 9-16, 2000.

JAKELAITIS, A.; SILVA, A.A.; SANTOS, J.B. \& VIVIAN, R. Qualidade da camada superficial de solo sob mata, pastagens e áreas cultivadas. Pesquisa Agropecuária Tropical, Goiânia, v.38, p.118- -127, 2008.

LiSBOA, B. B.; VARGAS, L. K.; SIlveirA, A. O. D.; MARTinS, A. F.; SElBACH, P. A. Indicadores microbianos de qualidade do solo em diferentes sistemas de manejo. Revista Brasileira de Ciência do Solo, Viçosa, G, v. 36, n. 1, p. 33-43, 2012.

LOSS, A.; PEREIRA, M. G.; SCHUlTZ, N.; ANJOS, L. H. C. D.; SILVA, E. M. R. D. Quantificação d carbono das substancias húmicas em diferentes sistemas de uso do solo e épocas de avaliação. Bragantia, Campinas, v. 69, n. 4, p. 913-922, 2010.

LOURENTE, E. R. P.; MERCANTE, F. M.; ALOVISI, A. M. T.; CEZESMUNDO FERREIRA GOMES; ADRIANO SOARES GASPARINI; NUNES, C. M. Atributos Microbiológicos, Químicos E Físicos De Solo Sob Diferentes Sistemas De Manejo E Condições De Cerrado. Pesquisa Agropecuá ria Tropical, Goiânia, v. 41, n. 1, p. 20 - 28, 2011.

MENDONÇA, E. D. S.; MATOS, E. D. S. Matéria Orgânica do solo: métodos de análises. Viçosa: UFV, 2005

NASCIMENTO, J. B.; CARVALHO, G. D.; CUNHA, E. Q.; FERREIRA, E. P. D. B.; LEANDRO, W. M.; DIDONET, A. Determinação da biomassa e atividade microbiana do solo sob cultivo orgânico do feijoeiro-comum em sistemas de plantio direto e convencional após cultivo de diferentes espécies de adubos verdes. Revista Brasileira de Agroecologia, Porto Alegre, v. 4, n. 2, 2009.

NEVES, C. M. N.; SILVA, M. L. N.; CURI, N.; MACEDO, R. L. G.; MOREIRA, F. M. D. S.; D'ANDRÉA, A. F. Indicadores biológicos da qualidade do solo em sistema agrossilvopastoril no nordeste do estado de minas gerais. Ciência e Agrotecnologia, Lavras, v. 33, n. 1, p. 105-112, 2009. 
PERES, J. G.; SOUZA, C.F.; LAVORENTI, N.A.; Avaliação dos efeitos da cobertura de palha de cana de açúcar na umidade e na perda de água no solo. Engenharia Agrícola, Jaboticabal,v. 30, n. 5, p. 875-886, 2010.

ROBOREDO, D., MAIA; J. C. de S.; OLIVEIRA, O. J.; ROQUE, C. G. Uso de dois penetrômetros na avaliação da resistência mecânica de um latossolo vermelho distrófico. Engenharia Agrícola, Jaboticabal, v.30, n.2, p. 307-314, 2010.

SILVA, I. R. D.; MENDONÇA, E. D. S. Matéria orgânica do solo. In: NOVAIS, R. F.; VICTOR, H. A. V..; BARROS, N. F.; FONTES, R. L. F.; CANTARUTTI NEVES, J. C. L. (Eds.). Fertilidade do Solo. Viçosa: Sociedade Brasileira de Ciência do Solo, 2007. 275-374.

SILVA, J. M.; ALBURQUERQUE, L. S. D.; SANTOS, T. M. C. D.; OliVEIRA, J. U. L. D.; GUEDES, E. L. F. Mineralização de vermicompostos estimada pela respiração microbiana. Revista Verde, Pombal, PB, v. 8, n. 4, p. 132-135, 2013.

SILVA, M. B.; KIEMANN, H. J.; SILVEIRA, P. M.; LANNA, A. C. Atributos biológicos do solo sob influência da cobertura vegetal e do sistema de manejo. Revista Pesquisa Agropecuária Brasileira, Brasília, v. 42, n.12, p. 1755-1761, 2007.

SILVA, R. R. D.; SILVA, M. L. N.; CARDOSO, E. L.; MOREIRA, F. M. D. S.; CURI, N.; ALIVISI, A. M. T. Biomassa e atividade microbiana em solos sob diferentes sistemas de manejo na região fisiográfica campos das vertentes - MG. Revista Brasileira de Ciência do Solo, Viçosa, MG, v. 34, p. 1585-1592, 2010.

UFV - UNIVERSIDADE FEDERAL DE VIÇOSA. SAEG - Sistema para análises estatísticas.Versão 9.1. Viçosa: Fundação Arthur Bernardes, UFV, 2007. 